\title{
SHARP INTEGRABILITY EXPONENTS AND CONSTANTS FOR MUCKENHOUPT AND GEHRING WEIGHTS AS SOLUTION TO A UNIQUE EQUATION
}

\author{
Arturo Popoli \\ Università degli Studi di Napoli "Federico II" \\ Dipartimento di Matematica e Applicazioni "R. Caccioppoli" \\ Via Cintia, 80126 Napoli, Italy; arturo.popoli@unina.it
}

\begin{abstract}
The sharp results for the self-improving and the transition properties of Gehring $R H_{q}$ and Muckenhoupt $A_{p}$ weights are unified and improved into corresponding sharp results for weights satisfying a general reverse Hölder inequality. We show that the optimal exponents of integrability as well as the best constants in the integral inequalities can be obtained by mean of the unique algebraic equation

$$
\left(\frac{x}{x-q}\right)^{\frac{1}{q}}=B\left(\frac{x}{x-p}\right)^{\frac{1}{p}}
$$

holding for the so called $B_{p}^{q}$ class (see 1.10) which contains the Gehring and Muckenhoupt classes as particular cases.
\end{abstract}

\section{Introduction}

A weight is a locally integrable function defined on a bounded interval $I \subset \mathbf{R}$ to $\left[0,+\infty\left[\right.\right.$. For a given exponent $p>1$ a weight $w$ is said to verify the $A_{p}$-condition if there exists a real constant $A>1$ such that, for every interval $J \subseteq I$, it holds

$$
f_{J} w\left(f_{J} w^{\frac{1}{1-p}}\right)^{p-1} \leq A .
$$

In the limit case $p=1$ the condition (1.1) becomes

$$
f_{J} w \leq A \underset{J}{\operatorname{essinf}} w
$$

and in this case we say that the weight $w$ verifies the $A_{1}$-condition.

The smallest constant $A$ verifying (1.1) or (1.2) is called the $A_{p}$-norm of the weight $w$ and is denoted by $A_{p}(w)$. On the other side, for a given fixed constant $A>1$, the Muckenhoupt class $A_{p}(A)$ is defined as the set of all weights $w$ verifying conditions (1.1) or (1.2), i.e. $A_{p}(w) \leq A$. These definitions have been introduced in 1972 by Muckenhoupt [16] to characterize those weights $w$ which make the maximal operator $M$ bounded on the weighted $L^{p}(w)$ space.

For a given exponent $q>1$ a weight $v$ is said to verify the $R H_{q}$-condition if there exists a real constant $G>1$ such that, for every interval $J \subseteq I$, it holds

$$
\left(f_{J} v^{q}\right)^{\frac{1}{q}} \leq G f_{J} v
$$

In the limit case $q=+\infty$ the condition (1.3) becomes

$$
\underset{J}{\operatorname{ess} \sup } v \leq G f_{J} v
$$

https://doi.org/10.5186/aasfm.2018.4351

2010 Mathematics Subject Classification: Primary 46E30; Secondary 26D15.

Key words: Reverse Hölder's inequalities, Muckenhoupt weights, Gehring lemma. 
and we say that the weight $v$ verifies the $R H_{\infty}$-condition.

The smallest constant $G$ verifying (1.3) or (1.4) is called the $R H_{q}$-norm of the weight $v$ and is denoted by $R H_{q}(v)$. For a given fixed constant $G>1$, the Gehring class $R H_{q}(G)$ is defined as the set of all weights $v$ verifying conditions (1.3) or (1.4), i.e. $R H_{q}(v) \leq G$. Such weights have been introduced in 1973 by Gehring [8] to prove a result of $L^{q}$-integrability of the gradient for quasi-conformal mappings.

As proved independently by Muckenhoupt and Gehring in their celebrated papers, these two classes both enjoy the remarkable self-improving property: for given couples of constants $p, A$ and $q, G$ defined as above, there exist limit exponents $p^{*}=p^{*}(p, A)<p$ and $q^{*}=q^{*}(q, G)>q$, and corresponding constants $D_{r}=D_{r}(p, A)$ and $C_{s}=C_{s}(q, A)$, such that the following inclusions hold true:

$$
\begin{gathered}
\left.\left.w \in A_{p}(A) \Longrightarrow w \in A_{r}\left(D_{r}\right) \quad \forall r \in\right] p^{*}, p\right], \\
v \in R H_{q}(G) \Longrightarrow v \in R H_{s}\left(C_{s}\right) \quad \forall s \in\left[q, q^{*}[.\right.
\end{gathered}
$$

The self-improving property has applications in the study of the optimal regularity of solutions to some elliptic PDE's (see for example Kenig [9] and Sbordone-Zecca [22]) where the $L^{p}$ solvability of the Dirichlet problem, $\operatorname{div} A(x) \nabla u=0$ on the unit disc $D$, with $u_{\mid \partial D}=\varphi$, can be expressed in terms of $R H_{q}$ conditions on the boundary $\partial D$ for the harmonic measures associated to $A(x)$, with $1 / p+1 / q=1$. Another recent application of the sharp results for the reverse Hölder inequalities can be found in Martio-Sbordone [15] in the study of $K$-quasiminimisers and their inverse.

In 1974 Coifman and Fefferman [5] proved the connection between the two classes: any Muckenhoupt weight belongs to some Gehring class and conversely. Namely, there exist exponents $s^{*}=s^{*}(p, A)$ and $r^{*}=r^{*}(q, G)$ and corresponding constants $C_{s}=C_{s}(p, A)$, and $D_{r}=D_{r}(q, G)$, such that the following transition properties hold true:

$$
\begin{aligned}
w \in A_{p}(A) & \Longrightarrow w \in R H_{s}\left(C_{s}\right) \quad \forall s<s^{*}, \\
v \in R H_{q}(G) & \Longrightarrow v \in A_{r}\left(D_{r}\right) \quad \forall r>r^{*} .
\end{aligned}
$$

These properties have been deeply investigated, particularly with respect to three problems:

(A) to find the value of the limit exponents $p^{*}$ and $q^{*}$ for which the self-improving property holds;

(B) to find the value of the limit exponents $r^{*}$ and $s^{*}$ for which the transition property holds;

(C) to find the best constants $C_{s}$ and $D_{r}$ for which the improved $A_{r}$-condition and the $\mathrm{RH}_{s}$-condition are satisfied.

There is an extensive literature on the matter and in dimension one most of the aspects have been addressed.

The first result goes back to 1990 and is due to D'Apuzzo and Sbordone [6] who found the optimal integrability exponent for monotonic $R H_{q}$ weights as solution of an algebraic equation. In 1992 Korenovskii [10] completely solved problem (A) by proving the corresponding results for $A_{p}$ weights with the significant improvement of removing the monotonicity assumptions.

For the limit class $A_{1}$ problems (B) and (C) have been resolved by Bojarski, Sbordone and Wik in [4] where both the sharp integrability exponent and the sharp constant have been found in explicit form. Their method has been applied to the class $R H_{\infty}$ by Basile, D'Apuzzo, Squillante in [1]. 
The complete solution to problem (B) has been provided by Malaksiano ([13], [14]) who found the sharp exponents for the transition from $A_{p}$ in $R H_{q}$ and viceversa, respectively in 2001 and 2002. The solution to problem (C) is due to Vasyunin [24] and Dindoš-Wall [7] who found the sharp constants for the self-improving and the transition properties respectively for $A_{p}$ and $R H_{q}$ weights.

With respect to these results there are some aspects we can point out:

i) definitions and notations are not yet uniformly consolidated, with some non trivial differences across the various papers;

ii) methods in the proofs are significantly different. For example the solution to problems (A) and (B) relies on some classical Hardy type inequalities and integral estimates while problem $(\mathrm{C})$ has required the use of the Bellman functions, which are a powerful tool to deal with optimization problems but require more technical complexity than the traditional methods;

iii) there are limited visible connections among the solutions, which have been provided in different forms and apparently require an ad hoc equation for every single result. As example, the best self-improving exponents for the $A_{p}$ weights appear in [24] and [10] as implicit solutions of two different equations.

Aim of this paper is to provide a unified solution to problems (A), (B) and (C) in a single and more general RHI Theorem which foresees the known results cited above as particular cases. More precisely, we show that the sharp exponents and the sharp constants can both be expressed as values of the $\omega$-function defined by

$$
\omega(p, q, x)=\left(\frac{x}{x-p}\right)^{-\frac{1}{p}}\left(\frac{x}{x-q}\right)^{\frac{1}{q}}
$$

provided the appropriate setting of variables.

The approach we follow is to consider the $A_{p}$ and $R H_{q}$ classes as special cases of the so called reverse Hölder's inequality (RHI) $B_{p}^{q}$ class: for given exponents $p$ and $q$ such that $p<q$, we say that the weight $u$ satisfies a reverse Hölder's inequality if there exists a constant $B$ such that, for any interval $J \subseteq I$, we have

$$
\left(f_{J} u^{q}\right)^{\frac{1}{q}} \leq B\left(f_{J} u^{p}\right)^{\frac{1}{p}}
$$

By recalling the classical Hölder's inequality it is clear that the definition is well posed only for $B \geq 1$, where the equality prevails in case of constant functions. The smallest constant, independent on the interval $J$, satisfying the inequality (1.10) is called the $B_{p}^{q}$-norm of the weight $u$ and will be denoted by $B_{p}^{q}(u)$; we refer to the inequality (1.10) as the $B_{p}^{q}$-condition for the weight $u$.

For a given fixed constant $B>1$ we denote with $B_{p}^{q}(B)$ the class of all weights $u$ on $I$ verifying the condition $(1.10)$ with constant $B$, i.e. $B_{p}^{q}(u) \leq B$. The $B_{p}^{q}$ classes have been investigated by several authors (see for example Bojarski [3], Sbordone [23], Wik [26], Korenovskii [11], Popoli [18], Vasyunin [25] and others) as they provide a natural generalization for the $A_{p}$ and the $R H_{q}$ classes and let us leverage some symmetries and properties of the $B_{p}^{q}$-characteristic equation

$$
\left(\frac{x}{x-q}\right)^{\frac{1}{q}}=B\left(\frac{x}{x-p}\right)^{\frac{1}{p}}
$$

which can be written in terms of $\omega$ function in the equivalent form

$$
\omega(p, q, x)=B .
$$


As far as we know, the equation (1.11) has been found for the first time in [18] (1997) to unify and extend the known sharp solutions to problem (A). In this paper we show that the characteristic equation (1.11) also solves problems (B) and (C) for one dimensional weights.

Actually, by observing that the $\omega$ function is strictly increasing for $x$ in $]-\infty, 0[$ and strictly decreasing in ]0, $+\infty[$, we have that the equation $\omega(p, q, x)=B$ admits only one negative solution $\nu_{-}=\nu_{-}(p, q, B)$ and one positive solution $\nu_{+}=\nu_{+}(p, q, B)$.

Our main result is the following RHI Theorem: exponents and best constants in problems (A) and (B) and (C) can all be expressed in terms of the two solutions $\nu_{-}$ and $\nu_{+}$of the equation (1.11).

Theorem 1.1. (RHI Theorem) Let $B>1$ and $p, q \in \mathbf{R}-\{0\}$ real numbers such that $p<q$. If $\nu_{-} \leq p$ and $\nu_{+} \geq q$ are the two solutions of the equation

$$
\omega(p, q, x)=B,
$$

then we have the following improvements of regularity:

(1) $u \in B_{p}^{q}(B) \Longrightarrow u \in B_{p}^{s}\left(C_{s}\right)$ for any $s \in \mathbf{R}^{+}$such that $q \leq s<q^{*}$ where $q^{*}=\nu_{+}(p, q, B)$ and

$$
C_{s}=\omega\left(p, s, q^{*}\right)
$$

(2) $u \in B_{p}^{q}(B) \Longrightarrow u \in B_{r}^{q}\left(D_{r}\right)$ for any $r \in \mathbf{R}^{-}$such that $p^{*}<r \leq p$ where $p^{*}=\nu_{-}(p, q, B)$ and

$$
D_{r}=\omega\left(r, q, p^{*}\right)
$$

The limit exponents $\nu_{+}$and $\nu_{-}$as well as the constants $C_{s}$ and $D_{r}$ are sharp. Moreover, statement (1) also holds true in the limit cases $p=-\infty$ and $p=0$ while statement (2) holds true also in the limit cases $q=0$ and $q=+\infty$.

Remark 1.2. The statement of the RHI Theorem clarifies the role of the $\omega$ function, which let us obtain the optimal ranges of integrability but also provides the sharp constants in the self-improved estimates, showing a precise link between exponents and constants.

This paper will proceed as follows. In Section 2 we provide notations and definitions, paying attention to the fact that in literature there is not yet a consolidated convention. In Section 3 we state the explicit version of the main RHI Theorem for the $A_{p}$ and $R H_{q}$ classes, to show how it works in the most interesting cases. In Section 4 we provide new simple proofs of the main known solutions to problems (A), (B) and (C) and show how all these theorems can be unified and derived by the main theorem. Moreover we derive a new property on the optimal integrability ranges from the link between exponents and constants. Finally Section 5 contains the proof of the main theorem.

\section{Definitions and notations}

Let $w$ be a weight. For a given exponent $p>1$ we define the $A_{p}$-norm of $w$ the following quantity

$$
A_{p}(w):=\sup _{J \subset I} f_{J} w\left(f_{J} w^{\frac{1}{1-p}}\right)^{p-1}
$$

where the supremum is taken over all intervals $J \subset I$. 
In the limit case $p=1$ the $A_{1}$-norm is defined by

$$
A_{1}(w):=\sup _{J \subset I} f_{J} w(\underset{J}{\operatorname{essinf}} w)^{-1}
$$

and for the limit case $p=\infty$ the $A_{\infty}$-norm is defined by

$$
A_{\infty}(w):=\sup _{J \subset I} f_{J} w \exp \left(f_{J} \log \frac{1}{w}\right)
$$

For $1 \leq p \leq \infty$ we say that $w$ is a Muckenhoupt $A_{p}$ weight if its $A_{p}$ norm is finite, i.e.

$$
w \in A_{p} \stackrel{\text { def }}{\Longleftrightarrow} A_{p}(w)<\infty .
$$

In other words the $A_{p}$-norm of a weight $w \in A_{p}$ represents the smallest constant for which the $A_{p}$-condition holds true independently on the interval $J \subseteq I$.

When we also fix a constant $A>1$ the couple of real numbers $(p, A)$ defines the $A_{p}$ Muckenhoupt class $A_{p}(A)$ :

$$
w \in A_{p}(A) \stackrel{\text { def }}{\Longleftrightarrow} A_{p}(w) \leq A
$$

and we will refer to $A$ as the $A_{p}$-constant of the class. Similarly, for a given exponent $q>1$ we define the $R H_{q}$-norm of $v$ as

$$
R H_{q}(v):=\sup _{J \subset I}\left(f_{J} v\right)^{-1}\left(f_{J} v^{q}\right)^{\frac{1}{q}}
$$

where the supremum, taken over all intervals $J \subset I$, represents the best constant for which the $R H_{q}$-condition holds true independently on the interval interval $J \subseteq I$. In the limit case $q=+\infty$ the $R H_{q}$-norm is defined as

$$
R H_{\infty}(v):=\sup _{J \subset I}\left(f_{J} v\right)^{-1} \operatorname{ess} \sup v .
$$

We say that $v$ is a Gehring $R H_{q}$ weight if its $R H_{q}$ norm is finite, i.e.

$$
v \in R H_{q} \stackrel{\text { def }}{\Longleftrightarrow} R H_{q}(v)<\infty .
$$

Again, if besides the exponent $q$ we also fix a constant $G>1$ it is well defined the Gehring class $\mathrm{RH}_{q}(G)$ by

$$
v \in R H_{q}(G) \stackrel{\text { def }}{\Longleftrightarrow} R H_{q}(v) \leq G
$$

and we will refer to $G$ as the $R H_{q}$-constant of the class. From the Hölder's inequality it is easy to observe that the $A_{p}$ and the $R H_{q}$ norms are respectively decreasing with respect to $p$ and increasing with respect to $q$ :

$$
\begin{aligned}
& r<p \Longrightarrow 1 \leq A_{p}(w) \leq A_{r}(w), \\
& q<s \Longrightarrow 1 \leq R H_{q}(v) \leq R H_{s}(v) .
\end{aligned}
$$

Hence $A_{r} \subset A_{p}$ and $R H_{s} \subset R H_{q}$ and it is natural to define $A_{\infty}=\bigcup_{p>1} A_{p}$.

Consistently, for given non zero exponents $p$ and $q$ such that $p<q$ we can introduce the quantity

$$
B_{p}^{q}(u):=\sup _{J \subset I}\left(f_{J} u^{p}\right)^{-\frac{1}{p}}\left(f_{J} u^{q}\right)^{\frac{1}{q}}
$$


as the $B_{p}^{q}$-norm of $u$ and say that $u$ is a $B_{p}^{q}$ weight if his $B_{p}^{q}$ norm is finite, i.e.

$$
u \in B_{q}^{p} \stackrel{\text { def }}{\Longleftrightarrow} B_{q}^{p}(u)<+\infty .
$$

Finally, if besides the exponents $p, q$ we also fix a constant $B>1$ we can define the RHI class $B_{p}^{q}(B)$ by

$$
u \in B_{q}^{p}(B) \stackrel{\text { def }}{\Longleftrightarrow} B_{q}^{p}(u) \leq B .
$$

It is immediate to observe how all the classes defined above can be seen as special cases of the $B_{p}^{q}$ reverse Hölder's inequality class:

(1) $A_{p}=B_{\frac{1}{1-p}}^{1}$

(2) $A_{1}=B_{-\infty}^{1}$

(3) $A_{\infty}=B_{0}^{1}$,

(4) $R H_{q}=B_{1}^{q}$,

(5) $R H_{\infty}=B_{1}^{+\infty}$.

with the special case $B_{-\infty}^{+\infty}$ corresponding to the Harnack inequality $\operatorname{ess}_{\sup } u \leq$ $B \operatorname{ess} \inf _{J} u$. In this paper we will see how the main theorem covers and unifies the known properties holding in the classes listed above.

Let us now recall the statement of the self-improving property for $A_{p}$ and $R H_{q}$ weights:

$$
\begin{aligned}
w \in A_{p}(A) \Longrightarrow & \text { there exist an exponent } r<p \\
& \text { and a constant } D>1: A_{p}(A) \subseteq A_{r}(D), \\
v \in R H_{q}(G) \Longrightarrow & \text { there exist an exponent } s<q \\
& \text { and a constant } C>1: R H_{q}(G) \subseteq R H_{s}(C) .
\end{aligned}
$$

Consequently, for the $A_{p}(A)$ and $R H_{q}(G)$ classes we can define

(1) the sharp self-improving exponents:

$$
\begin{aligned}
& \left.\left.p^{*}=p^{*}(p, A)=\operatorname{Inf}\{r \in] 1, p\right]: w \in A_{p}(A) \Longrightarrow w \in A_{r}\right\}, \\
& q^{*}=q^{*}(q, G)=\operatorname{Sup}\left\{s \in \left[q,+\infty\left[: v \in R H_{q}(G) \Longrightarrow v \in R H_{s}\right\},\right.\right.
\end{aligned}
$$

(2) the sharp self-improving constants:

$$
\begin{aligned}
D_{r} & \left.\left.=D_{r}(p, A)=\operatorname{Inf}\left\{D>1: A_{p}(A) \subseteq A_{r}(D)\right\} \quad \forall r \in\right] p^{*}, p\right], \\
C_{s}=C_{s}(q, G) & =\operatorname{Inf}\left\{C>1: R H_{q}(G) \subseteq R H_{s}(C)\right\} \quad \forall s \in\left[q, q^{*}[.\right.
\end{aligned}
$$

By fixing the $A_{p}$ and $R H_{q}$ constants (respectively set equal to $A$ and $G$ ), we can state the transition property which embeds an $A_{p}(A)$ class into a $R H_{q}(G)$ class and conversely:

$$
\begin{aligned}
w \in A_{p}(A) \Longrightarrow & \text { there exist an exponent } s>1 \\
& \text { and a constant } C>1: A_{p}(A) \subseteq R H_{s}(C), \\
v \in R H_{q}(G) \Longrightarrow & \text { there exist an exponent } r>1 \\
& \text { and a constant } D>1: R H_{q}(G) \subseteq A_{r}(D) .
\end{aligned}
$$

As for the self-improving property we introduce

(1) the sharp transition exponents:

$$
\begin{gathered}
s^{*}=s^{*}(p, A)=\operatorname{Sup}\left\{s>1: w \in A_{p}(A) \Longrightarrow w \in R H_{s}\right\}, \\
r^{*}=r^{*}(q, G)=\operatorname{Inf}\left\{r>1: v \in R H_{q}(G) \Longrightarrow v \in A_{r}\right\},
\end{gathered}
$$


(2) the sharp transition constants:

$$
\begin{aligned}
& C_{s}=C_{s}(p, A)=\operatorname{Inf}\left\{C>1: A_{p}(A) \subseteq R H_{s}(C)\right\} \quad \forall s<s^{*}, \\
& D_{r}=D_{r}(q, G)=\operatorname{Inf}\left\{C>1: R H_{q}(G) \subseteq A_{r}(D)\right\} \quad \forall r>r^{*} \text {. }
\end{aligned}
$$

\section{The $A_{p}$ and $R H_{q}$ theorems}

In this section we specialize the main RHI Theorem in two corollaries respectively for the $A_{p}$ and $R H_{q}$ classes, to show how the sharp results work in the most interesting cases.

Theorem 3.1. (Theorem $\boldsymbol{A}_{p}$ ) Let $A>1$ and $p \geq 1$ be real fixed numbers. Then if $\nu_{-}$and $\nu_{+}$are the two solutions to the equation

$$
\left(\frac{x}{x-1}\right)\left[\frac{(p-1) x}{1+(p-1) x}\right]^{p-1}=A
$$

we have the following sharp properties:

(1) $w \in A_{p}(A) \Longrightarrow w \in R H_{s}\left(C_{s}\right)$ for any s such that $1<s<s^{*}=\nu_{+}$and

$$
C_{s}=\left(\frac{\nu_{+}}{\nu_{+}-1}\right)^{-1}\left(\frac{\nu_{+}}{\nu_{+}-s}\right)^{\frac{1}{s}}
$$

(2) $w \in A_{p}(A) \Longrightarrow w \in A_{r}\left(D_{r}\right)$ for any $r$ such that $\left(1-\frac{1}{\nu_{-}}\right)=p^{*}<r$ and

$$
D_{r}=\left(\frac{\nu_{-}}{\nu_{-}-1}\right)\left[\frac{(r-1) \nu_{-}}{1+(r-1) \nu_{-}}\right]^{r-1}
$$

where the bounds $r *$ and $q *$ and the constants $C_{s}$ and $D_{r}$ cannot be improved. Moreover, statement (1) also holds true in the limit cases $p=1$ for $w \in A_{1}$ and $p=+\infty$ for $w \in A_{\infty}$.

Proof. It is sufficient to apply the RHI Theorem 1.1 to the class $A_{p}=B_{\frac{1}{1-p}}^{1}$ where the $A_{p}$ characteristic equation is $\omega\left(\frac{1}{1-p}, 1, x\right)=A$, which gives (3.1). In the limit case $p=1$ the characteristic equation for the class $A_{1}$ is $\omega(-\infty, 1, x)=A$ and the equation (3.1) becomes

$$
\frac{x}{x-1}=A
$$

which has a single (positive) solution $\nu_{+}=\frac{A}{A-1}$. In this case we can obtain the explicit value of the optimal constant in (3.2), that is

$$
C_{s}=\left[\frac{1}{A^{s-1}(A+s-A s)}\right]^{\frac{1}{s}} .
$$

In the limit case $p=+\infty$ the characteristic equation for the class $A_{\infty}$ is $\omega(0,1, x)=A$ and the equation (3.1) becomes

$$
\frac{x}{x-1} e^{-\frac{1}{x}}=A
$$

Theorem 3.2. (Theorem $\boldsymbol{R H}_{\boldsymbol{q}}$ ) Let $G$ and $q>1$ real fixed numbers. Then if $\nu_{-}$and $\nu_{+}$are the two solutions to the equation

$$
\left(\frac{x}{x-q}\right)^{\frac{1}{q}}=G \frac{x}{x-1}
$$

we have the following sharp properties: 
(1) $v \in R H_{q}(G) \Longrightarrow v \in R H_{s}\left(C_{s}\right)$ for any s such that $1<q \leq s<q^{*}=\nu_{+}$and

$$
C_{s}=\left(\frac{\nu_{+}}{\nu_{+}-1}\right)^{-1}\left(\frac{\nu_{+}}{\nu_{+}-s}\right)^{\frac{1}{s}}
$$

(2) $v \in R H_{q}(G) \Longrightarrow v \in A_{r}\left(D_{r}\right)$ for any $r$ such that $r>r^{*}=\left(1-\frac{1}{\nu_{-}}\right)$and

$$
D_{r}=\left(\frac{\nu_{-}}{\nu_{-}-1}\right)\left[\frac{(r-1) \nu_{-}}{1+(r-1) \nu_{-}}\right]^{r-1}
$$

where the bounds $s^{*}$ and $r^{*}$ and the constants $C_{s}$ and $D_{r}$ cannot be improved. Moreover, statement (2) holds also in the limit case $q=+\infty$ with $v \in R H_{\infty}$.

Proof. It is sufficient to apply the RHI Theorem 1.1 to the class $R H_{q}=B_{1}^{q}$ and note the $R H_{q}$ characteristic equation is given by $\omega(1, q, x)=G$ that gives (3.6). In the limit case $R H_{\infty}$ the left hand side of (3.6) tends to 1 as $q$ goes to $+\infty$ and the characteristic equation becomes

$$
G \frac{x}{x-1}=1
$$

which has the unique negative solution $\nu_{-}=\frac{1}{1-G}$. So statement (2) in the limit case says that $v \in R H_{q}(G) \Rightarrow v \in A_{r}\left(D_{r}\right)$ for any $r>G$ with the optimal constant given by

$$
D_{r}=\frac{1}{G}\left(\frac{r-1}{r-G}\right)^{r-1} .
$$

Remark 3.3. Let us point out the symmetry in the two theorems where the values of the optimal constants have now exactly the same expression thanks to the use of the $\omega$ function. This outcome is actually another aspect of the known duality properties and interactions holding between the two classes (see for example [20]).

\section{New proofs of sharp results in dimension one: a first consequence}

In this section we provide a simple proof of the main known sharp results in one-dimensional $A_{p}$ and $R H_{q}$ classes as corollaries of the $A_{p}$ and $R H_{q}$ Theorems. Furthermore we show a new aspect of the self-improving integrability property (Theorem 4.15).

Theorem 4.1. (D'Apuzzo, Sbordone, Korenovskii $[6,10]$ ) Let us fix $q>1$ and $G>1$. Then for every $v \in R H_{q}(G), v \in R H_{s}$ for any $s \in\left[q, q^{*}[\right.$ where the sharp exponent $q^{*}=q^{*}(q, G)$ is given by the unique positive solution of the equation

$$
\frac{x-1}{x}\left(\frac{x}{x-q}\right)^{1 / q}=G .
$$

Moreover, the value $q^{*}$ is sharp.

Proof. Part of statement (1) of the $R H_{q}$ Theorem.

Remark 4.2. Theorem 4.1 has been extended in [17] to the weighted $R H_{q}$ class. In particular it has been proved that the equation (4.1) does not change if we replace the Lebesgue measure with an absolutely continuos measure $d \mu$. Korenovskii extended this result to $B_{p}^{q}$ in [11]. 
Theorem 4.3. (Korenovskii [10]) Let us fix $p>1$ and $A>1$. Then for every $w \in A_{p}(A), w \in A_{r}$ for any $\left.\left.r \in\right] p^{*}, p\right]$ where the exponent $p^{*}=p^{*}(p, A)$ is the unique positive solution of the equation

$$
\frac{1}{y}\left(\frac{p-1}{p-y}\right)^{p-1}=A .
$$

Moreover, the value $p^{*}$ is sharp.

Proof. Immediate application of statement (2) of the $A_{p}$ Theorem where, by adopting the change of variable $x=\frac{1}{1-y}$, the equation (3.1) becomes (4.2).

Theorem 4.4. (Malaksiano [13]) Let us fix $q>1$ and $G>1$. Then, for every $r \in] r^{*},+\infty\left[\right.$ there exists a constant $D_{r}=D_{r}(q, G)$ such that the inclusion $R H_{q}(G) \subset$ $A_{r}\left(D_{r}\right)$ holds true and $r^{*}=r^{*}(q, G)$ is given by the positive root of the equation

$$
y\left(\frac{1}{1+q(y-1)}\right)^{\frac{1}{q}}=G
$$

and the value $r^{*}$ is optimal.

Proof. It is sufficient to apply statement (2) of the $R H_{q}$ Theorem where, by adopting the change of variable $x=\frac{1}{1-y}$, the equation (3.6) becomes (4.3).

Theorem 4.5. (Malaksiano [14]) Let us fix $p>1$ and $A>1$. Then, for every $s \in] 1, s^{*}\left[\right.$ there exists a constant $C_{s}=C_{s}(p, A)$ such that the inclusion $A_{p}(A) \subset$ $R H_{s}\left(C_{s}\right)$ holds true and $s^{*}=s^{*}(p, A)$ is given by the root of the equation

$$
\left(\frac{x(p-1)}{1+x(p-1)}\right)^{p-1} \frac{x}{x-1}=A
$$

and the value $s^{*}$ is optimal.

Proof. It is included in statement (1) of the $A_{p}$ Theorem where the equation (3.1) coincides with equation (4.4).

Theorem 4.6. (Bojarski, Sbordone, Wik [4]) Let $w$ be a function in $A_{1}(A)$. Then, for every $s<s^{*}=\frac{A}{A-1}$ and every interval $J \subseteq I$, it holds

$$
f_{J} w^{s} \leq \frac{1}{A^{s-1}(A+s-A s)}\left(f_{J} w\right)^{s}
$$

where the limit exponent $s^{*}$ and the right hand side constant in (4.5) are sharp.

Proof. By the $A_{p}$ Theorem 3.1 in the limit case $p=1$ we have $w \in A_{1}(A) \Rightarrow$ $w \in R H_{s}\left(C_{s}\right)$, i.e.

$$
\left(f_{J} w^{s}\right)^{\frac{1}{s}} \leq C_{s} f_{J} w
$$

for all intervals $J \subseteq I$ and for all $s<s^{*}$, where $s^{*}$ is the unique (positive) solution of the characteristic equation

$$
\frac{x}{x-1}=A
$$


which clearly gives $s^{*}=\frac{A}{A-1}$. By replacing the value of $s^{*}$ in the expression of the optimal constant (3.2) we obtain the optimal $R H_{s}$ constant

$$
C_{s}=\left[\frac{1}{A^{s-1}(A+s-A s)}\right]^{\frac{1}{s}}
$$

which easily leads to (4.5) by replacing $C_{s}$ in (4.6) and raising both members to $s$.

Theorem 4.7. (Basile, D'Apuzzo, Squillante [1]) Let $w$ be a function in $R H_{\infty}(G)$. Then, for every $r>r^{*}=G$ there exists a constant $D_{r}=D_{r}(A)$ such that the inclusion $R H_{\infty}(G) \subset A_{r}\left(D_{r}\right)$ where

$$
D_{r}=\frac{1}{G}\left(\frac{r-1}{r-G}\right)^{r-1}
$$

and the values $r^{*}$ and $D_{r}$ are optimal.

Proof. By the $R H_{q}$ Theorem 3.2, in the limit case $R H_{\infty}$ the left hand side of (3.6) tends to 1 as $q$ goes to $+\infty$ and the characteristic equation becomes $G \frac{x}{x-1}=1$ which has the unique negative solution

$$
\nu_{-}=\frac{1}{1-G}
$$

So, in the limit case, statement (2) of Theorem 3.2 says that $v \in R H_{q}(G)$ implies $v \in A_{r}\left(D_{r}\right)$ for any $r>G$ with the sharp constant (4.7) obtained by replacing the value of $\nu_{-}$in the expression (3.8).

Let us now state the main results to problem (C). The first one is part of Theorem 1 in [24] and provides the best range and the sharp constant of the transition property for $A_{p}$ weights:

Theorem 4.8. (Vasyunin [24]) Let $w \in A_{p}(A)$ with $A>1$ and $p>1$ real constants. If $s^{+}$is the positive solutions of the equation

$$
(1-t)\left(1-\frac{t}{p}\right)^{-p}=\frac{1}{A}
$$

then we have

$$
f_{J} w^{s} \leq C_{\max }(p, s, A)\left(f_{J} w\right)^{s}
$$

for any $s \in\left[1, \frac{p-s^{+}}{s^{+}(p-1)}[\right.$ and

$$
C_{\max }(p, s, A)=A^{\frac{1-s}{p}} \frac{\left(1-s^{+}\right)^{\gamma}}{1-\gamma s^{+}}
$$

where $\gamma=\frac{s p-s+1}{p}$. Moreover, the limit exponent and the constant $C_{\text {max }}$ are optimal.

Proof. Statement (1) in Theorem $A_{p}$ gives $w \in A_{p}(A) \Rightarrow w \in R H_{s}\left(C_{s}\right)$ for any $\mathrm{s}$ such that $1<s<s^{*}=\nu_{+}$, that is equivalent to (4.9) with $C_{s}=\left[C_{\max }\right]^{\frac{1}{s}}$ and

$$
s^{*}=\nu_{+}=\frac{p-s^{+}}{s^{+}(p-1)}
$$


which gives the relationship between the positive solutions of equations (4.8) and (3.1). Actually it can be verified that with the change of variables

$$
x=\frac{p-t}{t(p-1)}
$$

the characteristic equation (3.1) in Theorem $A_{p}$ becomes the equation (4.8). The optimal constant $C_{\max }\left(s^{+}\right)$can be obtained by replacing the expression of $\nu_{+}$in the constant $C_{s}\left(\nu_{+}\right)$in $(3.2)$. As

$$
C_{s}\left(\nu_{+}\right)=\left(\frac{\nu_{+}}{\nu_{+}-1}\right)^{-1}\left(\frac{\nu_{+}}{\nu_{+}-s}\right)^{\frac{1}{s}}=\frac{1}{A}\left[\frac{(p-1) \nu_{+}}{1+(p-1) \nu_{+}}\right]^{p-1}\left(\frac{\nu_{+}}{\nu_{+}-s}\right)^{\frac{1}{s}}
$$

we have that

$$
C_{\max }\left(s^{+}\right)=\left[C_{s}\left(\nu_{+}\right)\right]^{s}=\left[C_{s}\left(\frac{p-s^{+}}{s^{+}(p-1)}\right)\right]^{s}=\frac{1}{A^{s}}\left[1-\frac{s^{+}}{p}\right]^{p \gamma} \frac{1}{1-\gamma s^{+}}=\frac{A^{\gamma}}{A^{s}} \frac{\left[1-s^{+}\right]^{\gamma}}{1-\gamma s^{+}}
$$

that leads to (4.10), where the last equality is obtained by recalling that $s^{+}$solves (4.8)

Theorem 4.9. (Vasyunin [24]) Let $w \in A_{p}(A)$ with $A>1$ and $p>1$ real constants. If $s^{-}$is the negative solution to the equation

$$
(1-t)\left(1-\frac{t}{p}\right)^{-p}=\frac{1}{A}
$$

then we have $w \in A_{p}(A) \Longrightarrow w \in A_{r}$ for any $r$ such that $\frac{p\left(1-s^{-}\right)}{p-s^{-}}<r \leq p$ with

$$
A_{r}(w)=\frac{\left(1-\frac{s^{-}}{p}\right)^{r}}{\left(1-s^{-}\right)\left(1-\frac{r-p}{p(r-1)} s^{-}\right)^{r-1}} .
$$

Moreover, the limit exponents and the constants are optimal.

Proof. It can be achieved with the same approach of the previous theorem, by adopting the change of variables $x=\frac{p-t}{t(p-1)}$ to transform the characteristic equation (3.1) in Theorem $A_{p}$ into the equation (4.12). The relationship between the two negative solutions in this case is given by

$$
\frac{1}{1-p^{*}}=\nu_{-}=\frac{p-s^{-}}{s^{-}(p-1)}
$$

and to obtain the expressions of the optimal constant (4.13) it is sufficient to replace the values of the solutions according to the relationships above into (3.3). By setting $c=s^{-} / p$ the right hand expression in (4.13) can be simplified as

$$
A_{r}(w)=\frac{1-c}{1-p c}\left[\frac{1-c}{1-\frac{r-p}{r-1} c}\right]^{r-1}
$$

and can be obtained by replacing $\nu_{-}=\frac{1-c}{c(p-1)}$ into the expression of $D_{r}$ in Theorem 3.1 . 
Theorem 4.10. (Dindoš, Wall [7]) For any weight $v \in R H_{q}(G)$ with $G>1$ we have $v \in A_{r}\left(D_{r}(q, G)\right)$ for any $r$ such that $r>r^{*}$ where $r^{*}=r^{*}(q, \delta)$ is the unique positive solution to the equation

$$
\frac{(y / G)^{q}-1}{y-1}=q
$$

and

$$
D_{r}(q, G)=\frac{1}{r^{*}}\left(\frac{r-1}{r-r^{*}}\right)^{r-1}
$$

and the values $r^{*}$ and $D_{r}$ are optimal.

Proof. It is sufficient to apply statement (2) of the $R H_{q}$ Theorem where, by adopting the change of variable $x=\frac{1}{1-y}$, the equation (3.6) becomes (4.14).

Theorem 4.11. (Dindoš, Wall [7]) For any weight $v \in R H_{q}(G)$ with $G>1$ we have $v \in R H_{s}\left(C_{s}(q, G)\right)$ for any such that $q \leq s<q^{*}$ where $q^{*}$ is the unique positive solution to the equation

$$
\frac{x-q}{x}\left(\frac{G x}{x-1}\right)^{q}=1
$$

and

$$
C_{s}(q, G)=\frac{q^{*}-1}{q *}\left(\frac{q^{*}}{q^{*}-s}\right)^{\frac{1}{s}}
$$

and the values $q *$ and $C_{s}(q, G)$ are optimal.

Proof. The theorem coincides with statement (1) of the $R H_{q}$.

Remark 4.12. Note that in Theorems 4.8 and 4.9 the sharp results for the self-improving and the transition properties of for $A_{p}$ weights are found by mean of the two solutions (one negative and one positive) of the same equation, while the corresponding results for $R H_{q}$ weights in Theorems 4.10 and 4.11 are found by using the positive solutions of two different equations.

It remains to cover the important case of $A_{\infty}$. By using Theorem $A_{p}$ in the limiting case $p=+\infty$ we can state the following result.

Theorem 4.13. (Theorem $\boldsymbol{A}_{\infty}$ ) Let $A>1$. If $\nu_{+}$is the unique positive solution to the equation

$$
\frac{x}{x-1} e^{-\frac{1}{x}}=A,
$$

then $w \in A_{\infty}(A) \Longrightarrow w \in R H_{s}\left(C_{s}\right)$ for any $s$ such that $1<s<s^{*}=\nu_{+}$and

$$
C_{s}=\left(\frac{\nu_{+}}{\nu_{+}-1}\right)^{-1}\left(\frac{\nu_{+}}{\nu_{+}-s}\right)^{\frac{1}{s}} .
$$

Moreover, the limit exponent $s^{*}$ and the constant $C_{s}$ are sharp.

Proof. The theorem coincides with statement (1) of Theorem $A_{p}$ when $p=$ $+\infty$.

Remark 4.14. The sharp exponent as solution to the equation (4.18) can be found in Korenovskii and Fomichev [12]. A more complete result was proved by Vasyunin in [24] by mean of another equation which naturally provided a different 
expression for the best constant. Nevertheless, with appropriate change of variables, it can be seen that the constant in (4.19) and the corresponding one in [24] actually represent the same value.

Let us now state an interesting property that can be seen by using the link between exponents and constants via the function $\omega$. In essence, for a given class $R H_{q}(G)$, the limit exponent $q^{*}$ (depending on $q$ and $G$ ) defines an interval $\left[q, q^{*}[\right.$ where the Gehring condition works. This means that we can apply the self-improving property starting from any $s \in\left[q, q^{*}\right.$ [ and wonder whether the limit exponent $s^{*}$ for the class $R H_{s}\left(C_{s}\right)$ (depending on $s$ and $C_{s}$ ) has some relationship with $q^{*}$. Actually it can be proved that $s^{*}=q^{*}$. In other words, if we apply the self-improving property "moving forward" in the interval $\left[q, q^{*}[\right.$ we do not obtain a further improvement of integrability.

In the proof of the main RHI Theorem we will show independently that this is a general fact for $B_{p}^{q}$ weights. For now we think it is worth to highlight this property for $A_{p}$ and $R H_{q}$ weights as a consequence of Theorems 4.9 and 4.11. More precisely, the following theorem holds.

Theorem 4.15. Let us consider the classes $A_{p}(A)$ and $R H_{q}(G)$ and their corresponding sharp self-improving exponents $p^{*}(p, A)$ and $q^{*}(q, G)$. Then we have:

$$
\begin{array}{ll}
\forall s \in\left[q, q^{*}[\right. & s^{*}\left(s, C_{s}(q, G)\right)=q^{*}(q, G), \\
\left.\forall r \in] p^{*}, p\right] & r^{*}\left(r, D_{r}(p, A)\right)=p^{*}(p, A) .
\end{array}
$$

Proof. Let us prove the first identity. By Theorem 4.11 we have

$$
v \in R H_{q}(G) \Longrightarrow v \in R H_{s}\left(C_{s}\right) \quad \forall s \in\left[q, q^{*}[\right.
$$

where $q^{*}$ solves the equation (4.16). By recalling the definition of $\omega$ we can re-write (4.16) and (4.17) as follows:

$$
\frac{q^{*}-q}{q^{*}}\left(\frac{G q^{*}}{q^{*}-1}\right)^{q}=1 \Longleftrightarrow \omega\left(1, q, q^{*}\right)=G
$$

and

$$
C_{s}=\frac{q^{*}-1}{q^{*}}\left(\frac{q^{*}}{q^{*}-s}\right)^{\frac{1}{s}}=\omega\left(1, s, q^{*}\right)
$$

where the values $q^{*}$ and $C_{s}(q, G)$ are optimal. Now, for any $s$ in the admissible range of integrability $\left[q, q^{*}[\right.$, we can apply again the self-improving property and obtain

$$
v \in R H_{s}\left(C_{s}\right) \Longrightarrow v \in R H_{t}\left(C_{t}\right) \quad \forall t \in\left[s, s^{*}[\right.
$$

where $s^{*}$ as usual has to solve the equation

$$
\omega\left(1, s, s^{*}\right)=C_{s} .
$$

As $\omega(p, q, x)$ is strictly decreasing for positive values of $x$, the equation $\omega(1, s, x)=C_{s}$ admits just one positive solution in $x$. So, by comparing (4.22) and (4.23), we get

$$
q^{*}=s^{*}
$$

which is $(4.20)$.

The proof of (4.21) can be achieved exactly the same way by using Theorem 4.9.

Let us conclude this section with another important step on the way of unifying the theory of $A_{\infty}$ weights. It has been obtained by Korenovskii in [11] in the general 
case of weighted inequalities. We report here the version with the standard Lebesgue measure for sake of consistency.

Theorem 4.16. (Korenovskii [11]) Let $p, q \in \mathbf{R}-\{0\}$ with $p<q$ and assume that the non negative weight $u$ satisfies the $B_{p}^{q}(B)$ condition. For every $\gamma<\min \{0, p\}$ or $\gamma>\max \{0, q\}$ such that

$$
\left(1-\frac{p}{\gamma}\right)^{\frac{1}{p}}>B\left(1-\frac{q}{\gamma}\right)^{\frac{1}{q}}
$$

there exist positive real constants $B^{\prime}$ and $B^{\prime \prime}$ depending on $p, q, B$ and $\gamma$, such that

$$
\frac{1}{B^{\prime}}\left(f_{J} u^{q}\right)^{\frac{1}{q}} \leq\left(f_{J} u^{\gamma}\right)^{\frac{1}{\gamma}} \leq B^{\prime \prime}\left(f_{J} u^{p}\right)^{\frac{1}{p}}
$$

for every interval $J \subseteq I$, where if $\gamma$ does not satisfy the inequality 4.24 one of the two inequalities in 4.25 fails. More precisely the left inequality fails if $\gamma<p$ while the right one fails if $\gamma>q$.

\section{Proof of Theorem 1.1.}

Let us first recall some classic results that will be used to prove our main theorem.

Lemma 5.1. (Hardy's inequality) Let $u$ be a weight on an interval $[a, b]$ and $\alpha$ and $\beta$ real numbers such that either $1 \leq \alpha \leq \beta, \beta>1$, or $\alpha<0, \beta<0$. Then

$$
\int_{a}^{b}(x-a)^{\frac{\alpha}{\beta}-1} U^{\alpha}(x) d x \leq\left(\frac{\beta}{\beta-1}\right)^{\alpha} \int_{a}^{b}(x-a)^{\frac{\alpha}{\beta}-1} u^{\alpha}(x) d x
$$

where $U(x)=f_{a}^{x} u(t) d t$.

Lemma 5.2. (Hardy, Littlewood, Polya) Let $u$ be a nonincreasing weight on an interval $[a, b]$ and $\lambda \geq 1$ a real number. Then the following inequality holds:

$$
\left(\int_{a}^{b} u^{\lambda}(x) d x\right)^{\frac{1}{\lambda}} \leq \frac{1}{\lambda} \int_{a}^{b}(x-a)^{\frac{1}{\lambda}-1} u(x) d x
$$

Another key tool in the study of reverse Hölder inequalities are the equimeasurable properties of monotonic rearrangements, as far as we know applied for the first time by Sbordone in [21] and refined by Korenovskii in [10] and [11]. We need some preliminary definitions.

Let $\Phi$ be the class of positive convex functions and let us denote with $u_{*}$ and $u^{*}$ respectively the nonincreasing and nondecreasing rearrangements for the function $u$. The functions $u_{*}$ and $u^{*}$ are equimeasurble with $u$ in a set $E$ in the sense that for any real exponent $\lambda$ it holds

$$
\int_{E} u^{\lambda} d x=\int_{0}^{|E|}\left(u^{*}\right)^{\lambda}=\int_{0}^{|E|}\left(u_{*}\right)^{\lambda}
$$

It is well known that a convex function $\varphi \in \Phi$ verifies the so called Jensen inequality

$$
\varphi\left(f_{E} u(x) d x\right) \leq f_{E} \varphi(u(x)) d x
$$


This makes natural to define that a weight $u$ is said to verify the reverse Jensen inequality, that will be denoted by $u \in R J^{\varphi}(K)$, if there exists a real constant $K>1$ such that, for every interval $J \subseteq I$, it holds

$$
f_{J} \varphi(u(x)) d x \leq K \varphi\left(f_{J} u(x) d x\right) .
$$

The following theorem, proved by Korenovskii in 1992, provides the exact estimate for the equimeasurable rearrangements of weights verifying the Reverse Jensen Inequality:

Theorem 5.3. (Korenovskii [11]) Let $\varphi \in \Phi$ and $u \in R J^{\varphi}(K)$. Then

$$
\begin{aligned}
& f_{E} \varphi\left(u^{*}(x)\right) d x \leq K \varphi\left(f_{E} u^{*}(x) d x\right), \\
& f_{E} \varphi\left(u_{*}(x)\right) d x \leq K \varphi\left(f_{E} u_{*}(x) d x\right)
\end{aligned}
$$

with the same constant $K$ as in condition (5.5).

It is easy to observe that for $\varphi(t)=t^{q}$ or $\varphi(t)=t^{\frac{1}{1-p}}$ the reverse Jensen inequality corresponds respectively to the $R H_{q}$ and the $A_{p}$ conditions. Similarly for $\varphi(t)=t^{\frac{p}{q}}$ it becomes the $B_{p}^{q}$ condition. This means that theorem 5.3 will allow us to limit our proofs to the monotonic onedimensional weights to obtain the same results in the general case. For more details we refer to the book [11] where Theorem 5.3 is proved not only for the Lebesgue measure but in the general case of absolutely continuous measures.

The following lemma describes the known fact that the power type functions belong to $B_{p}^{q}$ and their sharp integrability exponents coincide with the sharp integrability exponents of the entire $B_{p}^{q}$ class they belong to.

Lemma 5.4. If $\nu_{-}$and $\nu_{+}$are the two roots of the equation $\omega(p, q, x)=B$, then the functions

$$
u_{ \pm}=x^{-\frac{1}{\nu_{ \pm}}}, \quad x \in[0,1]
$$

belong to $B_{p}^{q}(B)$ with constant $B=\omega\left(p, q, \nu_{ \pm}\right)$and we have:

(1) $u_{+} \in B_{p}^{s}$ iff $s<\nu_{+}$and $B_{p}^{\nu_{+}}\left(u_{+}\right)=+\infty$,

(2) $u_{-} \in B_{r}^{q}$ iff $r>\nu_{-}$and $B_{\nu_{-}}^{q}\left(u_{-}\right)=+\infty$.

Proof of this property can be found in most of the cited papers for the corresponding special case. For the $B_{p}^{q}$ class we refer to the book [11] (proof of Theorem C.8, pp. 181, 182).

Proof of RHI Theorem 1.1. Step 1. The first step is to prove the theorem for monotonic functions. Let us start proving Statement (1). Let $u \in B_{p}^{q}(B)$ be a non increasing weight on $[a, b]$, so that, for every $x \in[a, b]$

$$
\left(f_{a}^{x} u^{q}\right)^{\frac{1}{q}} \leq B\left(f_{a}^{x} u^{p}\right)^{\frac{1}{p}}
$$

From the $B_{p}^{q}$ condition we immediately have

$$
\int_{a}^{b}(x-a)^{\frac{q}{s}-1}\left(f_{a}^{x} u^{q}\right) d x \leq B^{q} \int_{a}^{b}(x-a)^{\frac{q}{s}-1}\left(f_{a}^{x} u^{p}\right)^{\frac{q}{p}} d x .
$$


If we set $\alpha=\frac{q}{p}, \beta=\frac{s}{p}$, we have $1<\alpha<\beta$ if $p>0$ and $\alpha<0, \beta<0$ if $p<0$. So Lemma 5.1 works and we can apply the Hardy's inequality to get

$$
\int_{a}^{b}(x-a)^{\frac{q}{s}-1}\left(f_{a}^{x} u^{p}\right)^{\frac{q}{p}} d x \leq\left(\frac{s}{s-p}\right)^{\frac{q}{p}} \int_{a}^{b}(x-a)^{\frac{q}{s}-1} u^{q} d x
$$

By combining (5.8) and (5.9) we easily get

$$
\int_{a}^{b}(x-a)^{\frac{q}{s}-1}\left(f_{a}^{x} u^{q}\right) d x \leq B^{q}\left(\frac{s}{s-p}\right)^{\frac{q}{p}} \int_{a}^{b}(x-a)^{\frac{q}{s}-1} u^{q} d x
$$

Thanks to Fubini's Theorem we can rewrite the integral on the left in (5.10) as follows

$$
\int_{a}^{b}(x-a)^{\frac{q}{s}-1}\left(f_{a}^{x} u^{q}\right) d x=\frac{s}{s-q}\left[\int_{a}^{b}(x-a)^{\frac{q}{s}-1} u^{q} d x-(b-a)^{\frac{q}{s}} f_{a}^{b} u^{q} d x\right]
$$

which yields

$$
\left[1-B^{q}\left(\frac{s-q}{s}\right)\left(\frac{s}{s-p}\right)^{\frac{q}{p}}\right] f_{a}^{b}(x-a)^{\frac{q}{s}-1} u^{q} d x \leq(b-a)^{\frac{q}{s}} f_{a}^{b} u^{q} d x .
$$

Let us now introduce the auxiliary function

$$
\chi_{q}(s)=\left[1-B^{q}\left(\frac{s-q}{s}\right)\left(\frac{s}{s-p}\right)^{\frac{q}{p}}\right]
$$

which can be written in terms of $\omega$ as

$$
\chi_{q}(s)=1-\left[B\left(\frac{s-q}{s}\right)^{\frac{1}{q}}\left(\frac{s}{s-p}\right)^{\frac{1}{p}}\right]^{q}=1-\left[\frac{B}{\omega(p, q, s)}\right]^{q} .
$$

Clearly $\chi_{q}(q)=1$ and, as $\omega(p, q, s)$ strictly decreases for positive values of $s$, the same does $\chi_{q}(s)$, which will be zero for a certain value $q^{*}>q$ given by the unique positive solution $\nu_{+}$to the equation $\frac{B}{\omega(p, q, x)}=1$. So $\chi_{q}\left(q^{*}\right)=0$ and

$$
\chi_{q}(s)>0 \Longleftrightarrow \frac{B}{\omega(p, q, s)}<1 \Longleftrightarrow\left(\frac{s-p}{s}\right)^{\frac{1}{p}}>B\left(\frac{s-q}{s}\right)^{\frac{1}{q}} .
$$

So we have that $\chi_{q}(s)>0$ in $\left[q, q^{*}[\right.$ and from (5.11) we can obtain

$$
\int_{a}^{b}(x-a)^{\frac{q}{s}-1} u^{q} d x \leq \frac{(b-a)^{\frac{q}{s}}}{\chi_{q}(s)} \int_{a}^{b} u^{q} d x .
$$

Let us now apply lemma 5.2 to the non increasing function $u^{q}$ for $\lambda=s / q>1$ which gives

$$
\left(\int_{a}^{b} u^{s} d x\right)^{\frac{q}{s}} \leq \frac{q}{s} \int_{a}^{b}(x-a)^{\frac{q}{s}-1} u^{q} d x .
$$

From the last two inequalities we can derive

$$
\left(\int_{a}^{b} u^{s} d x\right)^{\frac{q}{s}} \leq \frac{q}{s} \frac{(b-a)^{\frac{q}{s}}}{\chi_{q}(s)} \int_{a}^{b} u^{q} d x
$$

and, considering that $q>0$, we get to

$$
\left(f_{a}^{b} u^{s} d x\right)^{\frac{1}{s}} \leq\left[\frac{q}{s} \frac{1}{\chi_{q}(s)}\right]^{\frac{1}{q}}\left(f_{a}^{b} u^{q} d x\right)^{\frac{1}{q}} .
$$


Finally, setting $H_{s}=\left[\frac{q}{s} \frac{1}{\chi_{q}(s)}\right]^{\frac{1}{q}}$, by applying the $B_{p}^{q}(B)$ condition we conclude that

$$
\left(f_{a}^{b} u^{s} d x\right)^{\frac{1}{s}} \leq B H_{s}\left(f_{a}^{b} u^{p} d x\right)^{\frac{1}{p}}
$$

This means that $u$ belongs to $B_{p}^{s}\left(B H_{s}\right)$ for all values of $s$ such that $\chi(s)>0$ or, equivalently, for all $s \in\left[q, q^{*}\left[\right.\right.$ with $q^{*}=\nu_{+}(p, q, B)$ unique positive solution to the equation $\chi(x)=0$, i.e.

$$
\omega\left(p, q, q^{*}\right)=B
$$

where the value $q^{*}=\nu_{+}$cannot be improved (see Lemma 5.4). This proves the equation (1.12) and the first part of statement (1) in Theorem 1.1.

Nevertheless $B H_{s}$ is not yet the sharp constant. To find the best constant $C_{s} \leq$ $B H_{s}$ such that

$$
u \in B_{p}^{q}(B) \Longrightarrow u \in B_{p}^{s}\left(C_{s}\right) \quad \forall s \in\left[q, q^{*}[,\right.
$$

let us first observe that $H_{s}=1$ for $s=q$ and in (5.12) we obtain again the initial $B_{p}^{q}$ condition with constant $B$. So we have

$$
B \leq C_{s} \leq B H_{s} \quad \forall s \in\left[q, q^{*}\left[\Longrightarrow \lim _{s \rightarrow q} C_{s}=B .\right.\right.
$$

If now we pick an $s \in\left[q, q^{*}[\right.$ and apply again the self-improving property to the class $B_{p}^{s}\left(C_{s}\right)$ we obtain

$$
u \in B_{p}^{s}\left(C_{s}\right) \Longrightarrow u \in B_{p}^{t}\left(C_{t}\right) \quad \forall t \in\left[s, s^{*}[\right.
$$

where again $s^{*}$ is given by the unique positive solution of the characteristic equation, i.e.

$$
\omega\left(p, s, s^{*}\right)=C_{s}
$$

Now (5.14) works and we have

$$
B=\lim _{s \rightarrow q} C_{s}=\lim _{s \rightarrow q} \omega\left(p, s, s^{*}\right)=\omega\left(p, q, s^{*}\right) .
$$

By recalling that the equation $\omega(p, q, x)=B$ admits a unique positive solution in $x$, from (5.13) and (5.16) we get

$$
s^{*}=q^{*}
$$

(as independently proved in Theorem 4.15). By replacing in (5.15) we have $C_{s}=$ $\omega\left(p, s, q^{*}\right)$ which completes the proof of Statement (1) for finite values of $p$.

To study the limit case $B_{-\infty}^{q}$ when we ask $p$ to tend to $-\infty$, as $\lim _{p \rightarrow-\infty}\left(\frac{x}{x-p}\right)^{-\frac{1}{p}}=$ 1 we can define

$$
\omega(-\infty, q, x) \stackrel{\text { def }}{=} \lim _{p \rightarrow-\infty} \omega(p, q, x)=\lim _{p \rightarrow-\infty}\left(\frac{x}{x-p}\right)^{-\frac{1}{p}}\left(\frac{x}{x-q}\right)^{\frac{1}{q}}=\left(\frac{x}{x-q}\right)^{\frac{1}{q}}
$$

so the characteristic equation for $B_{-\infty}^{q}$ becomes

$$
\omega(-\infty, q, x)=\left(\frac{x}{x-q}\right)^{\frac{1}{q}}=B
$$

which has the unique positive solution

$$
\nu_{+}=\frac{q B^{q}}{B^{q}-1} .
$$


Note that in the case $q=1$, where $A_{1}=B_{-\infty}^{1}$, the characteristic equation is

$$
\omega(-\infty, 1, x)=\frac{x}{x-q}=B
$$

and the solution (5.18) coincides with the known solution $\nu_{+}=\frac{B}{B-1}$.

Finally in the limit case $p=0$ we can define

$$
\omega(0, q, x) \stackrel{\text { def }}{=} \lim _{p \rightarrow 0} \omega(p, q, x)=\lim _{p \rightarrow 0}\left(\frac{x}{x-p}\right)^{-\frac{1}{p}}\left(\frac{x}{x-q}\right)^{\frac{1}{q}}=e^{-\frac{1}{x}}\left(\frac{x}{x-q}\right)^{\frac{1}{q}}
$$

so that the characteristic equation for the class $B_{0}^{q}$ is given by

$$
\omega(0, q, x)=e^{-\frac{1}{x}}\left(\frac{x}{x-q}\right)^{\frac{1}{q}}=B
$$

which in the special case of $A_{\infty}=B_{0}^{1}$ becomes

$$
\omega(0,1, x)=e^{-\frac{1}{x}} \frac{x}{x-1}=B .
$$

Statement (2) can be proved the same way and assuming $u$ to be nondecreasing. Following similar steps as in statement 1 we can obtain

$$
\left[1-B^{-p}\left(\frac{r-p}{r}\right)\left(\frac{r}{r-q}\right)^{\frac{p}{q}}\right] f_{a}^{b}(x-a)^{\frac{p}{r}-1} u^{p} d x \leq(b-a)^{\frac{p}{r}} f_{a}^{b} u^{p} d x .
$$

As in previous case we introduce an auxiliary function by setting

$$
\psi_{p}(r)=\left[1-B^{-p}\left(\frac{r-p}{r}\right)\left(\frac{r}{r-q}\right)^{\frac{p}{q}}\right]
$$

that we write as

$$
\psi_{p}(r)=1-\left[\frac{1}{B}\left(\frac{r-p}{r}\right)^{\frac{1}{p}}\left(\frac{r}{r-q}\right)^{\frac{1}{q}}\right]^{p}=1-\left[\frac{\omega(p, q, r)}{B}\right]^{p} .
$$

Clearly it is $\psi_{p}(r)=1$ for $r=p$ and, as $\omega(p, q, r)$ strictly increases for negative values of $r$, the same does $\psi_{p}(r)$, which will be zero for a certain value $p^{*}<p$ given by the unique negative solution $\nu_{-}$to the equation $\frac{\omega(p, q, x)}{B}=1$. So $\psi_{q}\left(p^{*}\right)=0$ and

$$
\psi_{p}(r)>0 \Longleftrightarrow \frac{\omega(p, q, r)}{B}<1 \Longleftrightarrow\left(\frac{r-p}{r}\right)^{\frac{1}{p}}>B\left(\frac{r-q}{r}\right)^{\frac{1}{q}} .
$$

Hence, as $\psi_{p}(r)>0$ in $\left.] p^{*}, p\right]$, from (5.20) we can obtain

$$
\int_{a}^{b}(x-a)^{\frac{p}{r}-1} u^{p} d x \leq \frac{(b-a)^{\frac{p}{r}}}{\psi_{p}(r)} \int_{a}^{b} u^{p} d x .
$$

Now, being in this case $p<0$, lemma 5.2 works for the non increasing function $u^{p}$ and for $\lambda=r / p>1$ we have

$$
\left(\int_{a}^{b} u^{r} d x\right)^{\frac{p}{r}} \leq \frac{p}{r} \int_{a}^{b}(x-a)^{\frac{p}{r}-1} u^{p} d x
$$

which again, from the last two inequalities, yields

$$
\left(\int_{a}^{b} u^{r} d x\right)^{\frac{p}{r}} \leq \frac{p}{r} \frac{(b-a)^{\frac{p}{r}}}{\psi_{p}(r)} \int_{a}^{b} u^{p} d x
$$


Taking into account that $p<0$ we obtain

$$
\left[\frac{p}{r} \frac{1}{\psi_{p}(r)}\right]^{\frac{1}{p}}\left(f_{a}^{b} u^{p} d x\right)^{\frac{1}{p}} \leq\left(f_{a}^{b} u^{r} d x\right)^{\frac{1}{r}} .
$$

With the position $K_{r}=\left[\frac{p}{r} \frac{1}{\psi_{p}(r)}\right]^{\frac{1}{p}}$, by applying the $B_{p}^{q}(B)$ condition we conclude

$$
\left(f_{a}^{b} u^{q} d x\right)^{\frac{1}{q}} \leq \frac{B}{K_{r}}\left(f_{a}^{b} u^{r} d x\right)^{\frac{1}{r}}
$$

This means that $u$ belongs to $B_{r}^{q}\left(\frac{B}{K_{r}}\right)$ for all values of $r$ such that $\psi_{p}(r)>0$, or equivalently, for all $\left.r \in] p^{*}, p\right]$ where $p^{*}=\nu_{-}(p, q, B)$ is the unique negative solution of the equation $\psi_{p}(x)=0$, i.e.

$$
\omega\left(p, q, p^{*}\right)=B
$$

where the value $p^{*}=\nu_{-}$cannot be improved. This proves the first part of statement (2).

The best constant $D_{r} \leq \frac{B}{K_{r}}$ in $(5.21)$ can be obtain exactly the same way of statement (1): by applying the self-improving property in $B_{r}^{q}\left(D_{r}\right)$, with $r$ in the admissible interval $\left.] p^{*}, p\right]$, we can derive the equality $r^{*}=p^{*}$ and, thanks to the monotonicity of $\omega$, we can get to $D_{r}=\omega\left(r, q, p^{*}\right)$, which completes the proof of Statement (2) for finite values of $q$.

When we ask $q$ to tend to $+\infty$ in the limit case $B_{p}^{+\infty}$, as $\lim _{q \rightarrow+\infty}\left(\frac{x}{x-q}\right)^{\frac{1}{q}}=1$ we can define

$$
\omega(p,+\infty, x) \stackrel{\text { def }}{=} \lim _{q \rightarrow+\infty} \omega(p, q, x)=\lim _{q \rightarrow+\infty}\left(\frac{x}{x-p}\right)^{-\frac{1}{p}}\left(\frac{x}{x-q}\right)^{\frac{1}{q}}=\left(\frac{x}{x-p}\right)^{-\frac{1}{p}}
$$

so the characteristic equation for the class $B_{p}^{+\infty}$ becomes

$$
\omega(p,+\infty, x)=\left(\frac{x}{x-p}\right)^{-\frac{1}{p}}=B
$$

which has the unique negative solution

$$
\nu_{-}=\frac{p}{1-B^{p}} .
$$

When $p=1$ this case corresponds to the class $R H_{\infty}$ with the characteristic equation given by

and the known solution

$$
\omega(1,+\infty, x)=\frac{x-1}{x}=B
$$

$$
\nu_{-}=\frac{1}{1-B} .
$$

Finally, for the limit case $q=0$, we can define

$$
\omega(p, 0, x) \stackrel{\text { def }}{=} \lim _{q \rightarrow 0} \omega(p, q, x)=\lim _{q \rightarrow+\infty}\left(\frac{x}{x-p}\right)^{-\frac{1}{p}}\left(\frac{x}{x-q}\right)^{\frac{1}{q}}=\left(\frac{x}{x-p}\right)^{-\frac{1}{p}} e^{\frac{1}{x}}
$$

so that the characteristic equation for the class $B_{p}^{0}$ is given by

$$
\omega(p, 0, x)=\left(\frac{x}{x-p}\right)^{-\frac{1}{p}} e^{\frac{1}{x}}=B
$$


which completes the proof of Statement (2) also for the limit cases.

Step 2. Removing the monotonicity assumptions. Finally, to remove the monotonicity assumptions we recall the properties of equimeasurable rearrangements as stated in Theorem 5.3. For sake of simplicity we do not indicate the steps to apply this standard argument that has been used the first time by Korenovskii in [10] and can be found in details in many papers (Korenovskii [11], Popoli [18], Malaksiano [13] and [14], and others).

The proof is now completed.

Acknowledgment. The author wishes to thank the anonymous referee for his/her valuable comments on the first version of this paper.

\section{References}

[1] Basile, L., L. D'Apuzzo, and M. Squillante: The limit class of Gehring type $G_{\infty}$. - Boll. Unione Mat. Ital. 11:B, 1997, 871-884.

[2] Beznosova, O., and A. Reznikov: Sharp estimates involving $A_{\infty}$ and $L \log L$ constants, and their applications to PDE. - St. Petersburg Math. J. 26:1, 2015, $27-47$.

[3] Bojarski, B.: Remarks on the stability of inverse Hölder inequalities and quasiconformal mappings. - Ann. Acad. Sci. Fenn. Math. 10, 1985, 89-94.

[4] Bojarski, B., C. Sbordone, and I. Wik: The Muckenhoupt class $A_{1}(\mathbf{R})$. - Studia Math. 101:2, 1992, 155-163.

[5] Coifman, R. R., and C. Fefferman: Weighted norm inequalities for maximal functions and singular integrals. - Studia Math. 51, 1974, 241-250.

[6] D'Apuzzo, L., and C. Sbordone: Reverse Hölder inequalities: a sharp result. - Rend. Mat. Appl. (VII) 10, 1990, 357-366.

[7] Dindoš, M., and T. Wall: The sharp $A_{p}$ constant for weights in a reverse Hölder class. Rev. Mat. Iberoam. 25:2, 2009, 559-594.

[8] Gehring, F. W.: The $L^{p}$-integrability of the partial derivatives of a quasiconformal mapping. - Acta Math. 130, 1973, 265-277.

[9] Kenig, C. E.: Harmonic analysis techniques for second order elliptic boundary value problems. - Conference Board of Mathematical Science 83, Amer. Math. Soc., Providence, 1991.

[10] KorenovskiI, A. A.: The exact continuation of a reverse Hölder inequality and Muckenhoupt's conditions - Math. Notes 52, 1992, 1192-1201.

[11] Korenovskir, A. A.: Mean oscillations and equimeasurable rearrangements of functions. Lect. Notes Unione Mat. Ital. 4. Springer, Berlin; UMI, Bologna, 2007.

[12] Korenovskit, A. A., and Fomichev, V.V.: Self-improvement of summability factors of functions satisfying the reverse Hölder inequality in limit cases. - Ukrainian Math. J. 62:4, $2010,552-563$.

[13] Malaksiano, N. A.: The exact inclusions of Gehring classes in Muckenhoupt classes. - Mat. Zametki 70:, 2001, 742-750 (in Russian); English transl. in Math. Notes 70:5-6, 2001, 673-681.

[14] Malaksiano, N. A.: The precise embeddings of one-dimensional Muckenhoupt classes in Gehring classes. - Acta Sci. Math. 68:1-2, 2002, 237-248.

[15] Martio, O. and C. Sbordone: Quasiminimizers in one dimension: integrability of the derivate, inverse function and obstacle problems. - Ann. Mat. Pura Appl. 186, 2007, 579-590.

[16] Muckenhoupt, B.: Weighted norm inequalities for the Hardy maximal function. - Trans. Amer. Math. Soc. 165, 1972, 207-226.

[17] Popoli, A.: Weighted reverse Hölder inequalities. - Rend. Accad. Sci. Fis. Mat. Napoli 62, $1995,187-211$. 
[18] Popoli, A.: Optimal integrability in $B_{p}^{q}$ classes. - Le Matematiche (Catania), 52:I, 1997, $159-170$.

[19] Popoli, A.: On an integral inequality and related summability results. - Anal. Math. 29, 2003, 135-145.

[20] Popoli, A.; C. Sbordone, and G. Zecca: Sharp interactions among $A_{\infty}$ weights on the real line. - Ric. Mat. 64:2, 2015, 289-301.

[21] Sbordone, C.: Rearrangement of functions and reverse Hölder inequalities. - Ennio De Giorgi colloquium (Paris, 1983), Res. Notes in Math. 125, Pitman, Boston, MA, 1985, 139-148.

[22] Sbordone, C., and G. Zecca: The $L^{p}$-solvability of the Dirichlet problem for planar elliptic equations, sharp results. - J. Fourier Anal. Appl. 15, 2009, 871-903.

[23] Sbordone, C.: Sharp embeddings for classes of weights and applications - Rend. Accad. Naz. Sci. XL Mem. Mat. Appl. 29:1, 2005, 339-354.

[24] Vasyunin, V.: The exact constant in the inverse Hölder inequality for Muckenhoupt weights. - Algebra i Analiz 15:1, 2003, 73-117 (in Russian); English transl. in St. Petersburg Math. J. 15:1, 2004, 49-79.

[25] Vasyunin, V.: Mutual estimates of $L^{p}$-norms and the Bellman function. - J. Math. Sci. 156:5, 2009; transl. from Zap. Nauchn. Sem. S.-Peterburg. Otdel. Mat. Inst. Steklov. (POMI) 355, 2008, 81-138.

[26] WiK, I.: On Muckenhoupt's classes of weight functions. - Studia Math. 94, 1989, 245-255.

Received 24 July 2017 • Accepted 1 February 2018 